\title{
Effects of metformin on hyperglycemia in an experimental model of tacrolimus- and sirolimus- induced diabetic rats
}

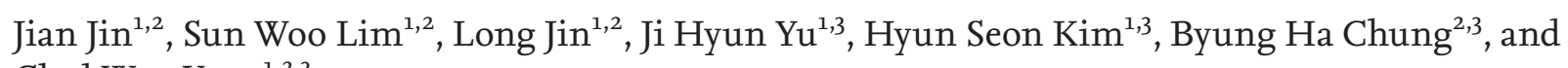
Chul Woo Yang ${ }^{1,2,3}$

\begin{abstract}
${ }^{1}$ Transplant Research Center, ${ }^{2}$ Convergent Research Consortium for Immunologic Disease, ${ }^{3}$ Division of Nephrology, Department of Internal Medicine, College of Medicine, Seoul St. Mary's Hospital, The Catholic University of Korea, Seoul, Korea
\end{abstract}

Received: December 8, 2015

Revised : February 3, 2016

Accepted: February 9, 2016

\section{Correspondence to}

Chul Woo Yang, M.D.

Division of Nephrology,

Department of Internal

Medicine, College of Medicine,

Seoul St. Mary's Hospital, The

Catholic University of Korea, 222

Banpo-daero, Seocho-gu, Seoul

06591, Korea

Tel: $+82-2-2258-6851$

Fax: +82-2-536-0323

E-mail: yangch@catholic.ac.kr
Background/Aims: Metformin (MET) is a first-line drug for type 2 diabetes mellitus (DM); its effect on new-onset diabetes after transplantation caused by immunosuppressant therapy is unclear. We compared the effects of MET on DM caused by tacrolimus (TAC) or sirolimus (SRL).

Methods: DM was induced by injection of TAC $(1.5 \mathrm{mg} / \mathrm{kg})$ or SRL $(0.3 \mathrm{mg} / \mathrm{kg})$ for 2 weeks in rats, and MET (200 mg/kg) was injected for 2 more weeks. The effects of MET on DM caused by TAC or SRL were evaluated using an intraperitoneal glucose tolerance test (IPGTT) and by measuring plasma insulin concentration, islet size, and glucose-stimulated insulin secretion (GSIS). The effects of MET on the expression of adenosine monophosphate-activated protein kinase (AMPK), a pharmacological target of MET, were compared between TAC- and SRL-treated islets.

Results: IPGTT showed that both TAC and SRL induced hyperglycemia and reduced plasma insulin concentration compared with vehicle. These changes were reversed by addition of MET to SRL but not to TAC. Pancreatic islet cell size was decreased by TAC but not by SRL, but addition of MET did not affect pancreatic islet cell size in either group. MET significantly increased GSIS in SRL- but not in TAC-treated rats. AMPK expression was not affected by TAC but was significantly decreased in SRL-treated islets. Addition of MET restored AMPK expression in SRL-treated islets but not in TAC-treated islets.

Conclusions: MET has different effects on hyperglycemia caused by TAC and SRL. The discrepancy between these drugs is related to their different mechanisms causing DM.

Keywords: New onset diabetes after transplantation; Tacrolimus; Sirolimus; Metformin

\section{INTRODUCTION}

New-onset diabetes after transplantation (NODAT) is a serious complication after kidney transplantation (KT) and is associated with worse patient and graft survival [1-3]. The causes of NODAT are multifactorial. Among them, the relationship between NODAT and immunosuppressive medications used after transplantation has been well documented [4], and immunosuppressive drugs account for up to $74 \%$ of the risk of NODAT development $[5,6]$.

Tacrolimus (TAC) and cyclosporine are the mainstay 
of immunosuppressive therapy in transplantation [7]. However, long-term treatment with calcineurin inhibitors (CNIs) causes both irreversible and chronic renal dysfunction as well as diabetes and cardiovascular disease in KT recipients [8]. Sirolimus (SRL), a mammalian target of rapamycin inhibitor, is a promising immunosuppressant for reducing or replacing CNIs [9]. However, more recent data from the US Renal Data System suggest that SRL is also independently associated with an increased risk of NODAT in KT recipients [10,11].

Metformin (MET), an oral hypoglycemic agent, is often prescribed to prevent or treat type 2 diabetes in people who have not undergone transplantation [12]. There is increasing interest in the use of MET after solid organ transplantation [13]. The safety and efficacy of MET in patients with preexisting diabetes who undergo transplantation or who develop NODAT have been demonstrated [14]. However, the effects of MET on controlling NODAT in KT recipients receiving CNIs or SRL have not been well studied. Therefore, we aimed to study whether MET can effectively control hyperglycemia caused by immunosuppressant therapy, specifically TAC and SRL.

\section{METHODS}

\section{Animal experiments}

The Animal Care Committee at the Catholic University of Korea approved the experimental protocol (CUMC2013-0131-03). Male Sprague Dawley rats (Charles River Technology, Seoul, Korea) weighing 200 to $220 \mathrm{~g}$ were housed in individual cases in a temperature- and light-controlled environment and allowed free access to a low-salt diet (0.05\% sodium; Teklad Premier, Madison, WI, USA) and tap water.

The experimental design is shown in Fig. 1. Rats were randomized to five groups ( $\mathrm{n}=9$ per group) as follows. (1) The vehicle group $(\mathrm{VH})$ received olive oil (Sigma, St. Louis, MO, USA) daily via oral gavage for 4 weeks; (2) the TAC group received TAC $(1.5 \mathrm{mg} / \mathrm{kg}$; Prograf, Astellas Pharma, Ibaraki, Japan) injected daily subcutaneously for 4 weeks; (3) the SRL group received SRL (0.3 mg/ kg; Wyeth-Ayerst Research, Princeton, NJ, USA) injected daily subcutaneously for 4 weeks; (4) the TAC + MET group received TAC injected daily for 4 weeks and MET (200 mg/kg; Dongwha Pharmaceutical Co., Seoul, Korea) daily via oral gavage from day 14; and (5) the SRL + MET group received SRL injected daily for 4 weeks and MET daily via oral gavage from day 14. An intraperitoneal glucose tolerance test (IPGTT) was performed at the end of the 4-week treatment period.

In this study, we chose $1.5 \mathrm{mg} / \mathrm{kg}$ of TAC and $0.3 \mathrm{mg} / \mathrm{kg}$ of SRL based on our previous study which showed dose-dependent increase of blood glucose level by IPGTT $[15,16]$. MET dose $(200 \mathrm{mg} / \mathrm{kg})$ was chosen based on previous reports that the MET doses of 100 and $200 \mathrm{mg} / \mathrm{kg}$ are effective in glucose control [17-19] and MET at doses $\geq$ $600 \mathrm{mg} / \mathrm{kg} /$ day shows clinical signs of toxicity [20]. Thus, the dose of MET used in this study is suitable for blood glucose control without MET toxicity.

Rats were pair-fed and their body weight was monitored daily. After the 4-week treatment period, animals were housed individually in metabolic cages (Tecniplast, Gazzada, Italy), and their water intake and urine volume were measured over 24 hours. On the following day, animals were anesthetized, and blood samples and tissue specimens were obtained for further analysis.

\section{Intraperitoneal glucose tolerance test}

The IPGT'T was performed at the end of the 4-week treatment period as previously described [21]. Briefly, after 1 day of fasting, $25 \%$ dextrose $(2 \mathrm{~g} / \mathrm{kg}$ ) was injected, and the blood glucose concentration was measured just before and 30, 60, 90, and 120 minutes after the injection using a glucose analyzer (Accu-Chek, Roche Diagnostics, Basel, Switzerland). The area under the curve for glucose (AUCg) was calculated by trapezoidal estimation from the values obtained in the IPGTTT.

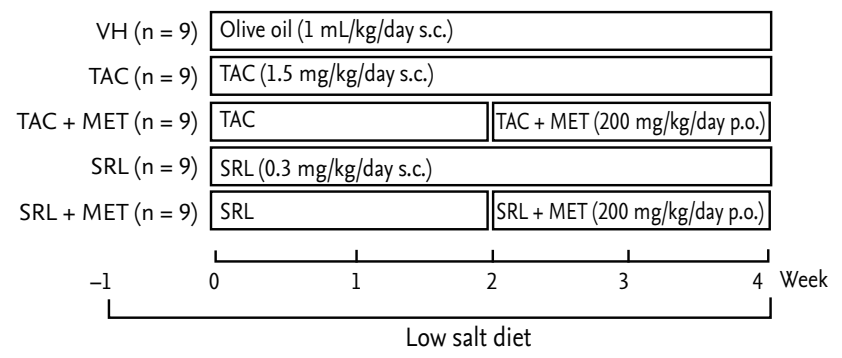

Figure 1. Experimental design for this study. After a lowsalt diet for 1 week, animals were divided into five groups of nine rats each and were treated with vehicle group (VH), tacrolimus (TAC), sirolimus (SRL), or TAC or SRL plus metformin (MET). S.C., subcutaneous; P.O., oral gavage. 


\section{Measurement of serum creatinine concentration and trough levels of TAC and SRL}

Serum creatinine concentration was measured using a quantitative enzyme colorimetric method (Stanbio Laboratory, Boerne, TX, USA). The whole-blood TAC and SRL concentrations were quantified using liquid chromatography-mass spectrometry with an API3000 LC/ MS/MS system (Applied Biosystems, Foster City, CA, USA) equipped with an electrospray ionization interface used to generate negative ions $[22,23]$.

\section{Measurement of insulin concentration}

Insulin concentration was measured in plasma from experimental animals and in culture media from islet cells subjected to a glucose-stimulated insulin secretion (GSIS) test. Insulin concentration was measured using a rat insulin sandwich enzyme-linked immunosorbent assay (ELISA) kit according to the manufacturer's protocol (Millipore Corp., St. Charles, MO, USA). Absorbance was measured at $450 \mathrm{~nm}$ using a Versa Max ELISA Microplate Reader (Molecular Devices, Sunnyvale, CA, USA).

\section{Measurement of pancreatic islet size}

To detect insulin, mounted tissue sections were incubated overnight at $4^{\circ} \mathrm{C}$ with polyclonal guinea pig anti-insulin antibody (1:3,000; Invitrogen, Carlsbad, CA, USA) as the primary antibody. The sections were incubated with donkey antiguinea pig antibody (1:200; Jackson ImmunoResearch Laboratories, West Grove, PA, USA) as the secondary antibody. The area per pancreas section occupied by insulin staining was calculated for each pancreas using an image analyzer (TDI Scope Eye version 3.5, Olympus, Seoul, Korea).

\section{In vitro study of GSIS}

To measure glucose-stimulated insulin release, isolated islets were treated initially with TAC $(30 \mathrm{ng} / \mathrm{mL})$ or SRL (90 ng/mL) for 12 hours, followed by treatment with MET ( $165 \mathrm{ng} / \mathrm{mL})$ for a further 12 hours. The islets were washed with Krebs-Ringer Modified Buffer (KRB; $130 \mathrm{mmol} / \mathrm{L} \mathrm{NaCl}, 3.6 \mathrm{mmol} / \mathrm{L} \mathrm{KCl}, 1.5 \mathrm{mmol} / \mathrm{L} \mathrm{CaCl}_{2}$, $0.5 \mathrm{mmol} / \mathrm{L} \mathrm{MgSO}_{4}, 0.5 \mathrm{mmol} / \mathrm{L} \mathrm{KH}_{2} \mathrm{PO}_{4}, 2.0 \mathrm{mmol} / \mathrm{L}$ $\mathrm{NaHCO}_{3}$, and $10 \mathrm{mmol} / \mathrm{L}$ HEPES), and the supernatant was collected (basal) and replaced with KRB containing $25 \mathrm{mM}$ glucose for an additional 30 minutes. The super- natant was sampled to measure the insulin level. Fig. 2 shows the workflow for the isolation of islets from rats.

\section{Immunoblot analysis of adenosine monophosphate- activated protein kinase expression}

To identify the expression of adenosine monophosphate-activated protein kinase (AMPK), isolated rat islets were treated initially with TAC (30 ng/mL) or SRL (90 $\mathrm{ng} / \mathrm{mL}$ ) for 12 hours, followed by treatment with MET (165 ng/mL) for a further 12 hours. After washing with $\mathrm{KRB}$, the islets were pelleted by centrifugation, and lysis buffer was added (10 mM Tris, pH 7.5, 1\% SDS, 1 mM $\mathrm{NaVO}_{4}$ ). The blots which transferred equal amount of protein resolves were incubated with blocking solution containing $5 \%$ nonfat milk (BD Difco, Sparks, MD, USA) in phosphate buffered saline, followed by an overnight incubation at $4^{\circ} \mathrm{C}$ with polyclonal rabbit antitotal AMPK
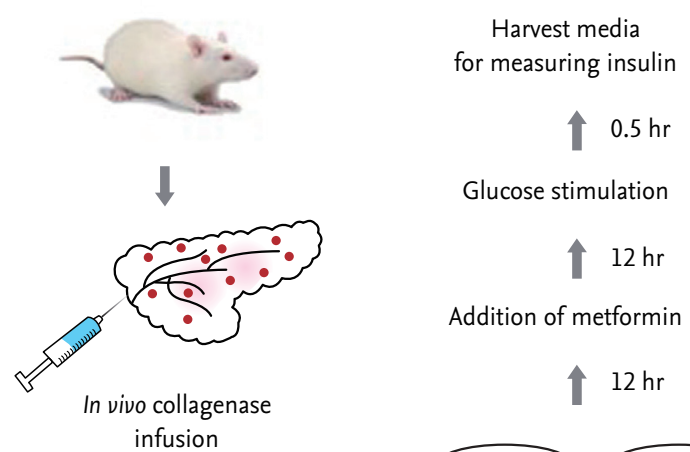

Glucose stimulation

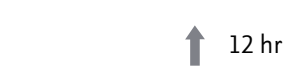

Addition of metformin

vivo collagenase

$12 \mathrm{hr}$
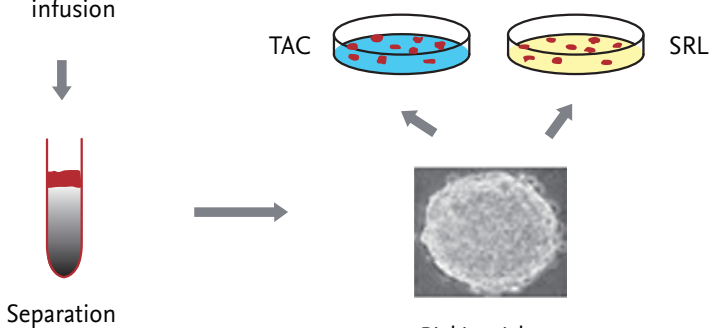

Picking islets

Figure 2. Schematic workflow for the isolation of islets from rats and the glucose-stimulated insulin secretion test. Rat pancreatic islets were isolated from rats by digesting the pancreatic duct with collagenase P. After digestion, the islets were separated on Histopaque 1077 (Sigma). Islets were picked up with a glass loop under a dissecting microscope. Islets were preincubated in conditioning media for 1 day. On the next day, islets were initially exposed to tacrolimus (TAC; $30 \mathrm{ng} / \mathrm{mL}$ ) or sirolimus (SRL; $90 \mathrm{ng} / \mathrm{mL}$ ) for 12 hours, followed by addition of metformin $(165 \mathrm{ng} / \mathrm{mL})$ for a further 12 hours. At the end of the incubation period, islets were incubated for 30 minutes in $25 \mathrm{mM}$ glucose, and the supernatant fluid was sampled and insulin concentration measured. 
Table 1. Effect of metformin on basic parameters in each group

\begin{tabular}{lccccc}
\hline Parameter & VH & TAC & TAC + MET & SRL & SRL + MET \\
\hline$\Delta$ BW, g & $75 \pm 6$ & $50 \pm 5^{\mathrm{a}}$ & $46 \pm 6^{\mathrm{a}}$ & $46 \pm 2^{\mathrm{a}}$ & $5^{2} \pm 2^{\mathrm{a}}$ \\
Water intake, mL/day & $26 \pm 4$ & $38 \pm 3^{\mathrm{a}}$ & $28 \pm 1^{\mathrm{b}}$ & $25 \pm 2^{\mathrm{a}}$ & $17 \pm 1^{\mathrm{a}, \mathrm{b}}$ \\
Urine volume, $\mathrm{mL} /$ day & $26 \pm 4$ & $31 \pm 3^{\mathrm{a}}$ & $21 \pm 2^{\mathrm{b}}$ & $27 \pm 4^{\mathrm{a}}$ & $12 \pm 1^{\mathrm{b}}$ \\
Serum creatinine, $\mathrm{mg} / \mathrm{dL}$ & $0.35 \pm 0.02$ & $0.76 \pm 0.04^{\mathrm{a}}$ & $0.56 \pm 0.07^{\mathrm{a}, \mathrm{b}}$ & $0.36 \pm 0.01$ & $0.38 \pm 0.02$ \\
Trough level, ng/mL & - & $10.2 \pm 1.3$ & $10.7 \pm 0.9$ & $3.2 \pm 0.3$ & $2.6 \pm 0.2$ \\
\hline
\end{tabular}

Values are presented as means \pm standard error.

$\mathrm{VH}$, vehicle group; TAC, tacrolimus; MET, metformin; SRL, sirolimus; $\Delta \mathrm{BW}$, changes of body weight.

${ }^{\mathrm{a}} \mathrm{p}<0.05$ vs. $\mathrm{VH},{ }^{\mathrm{b}} \mathrm{p}<0.05$ vs. the corresponding immunosuppressants.
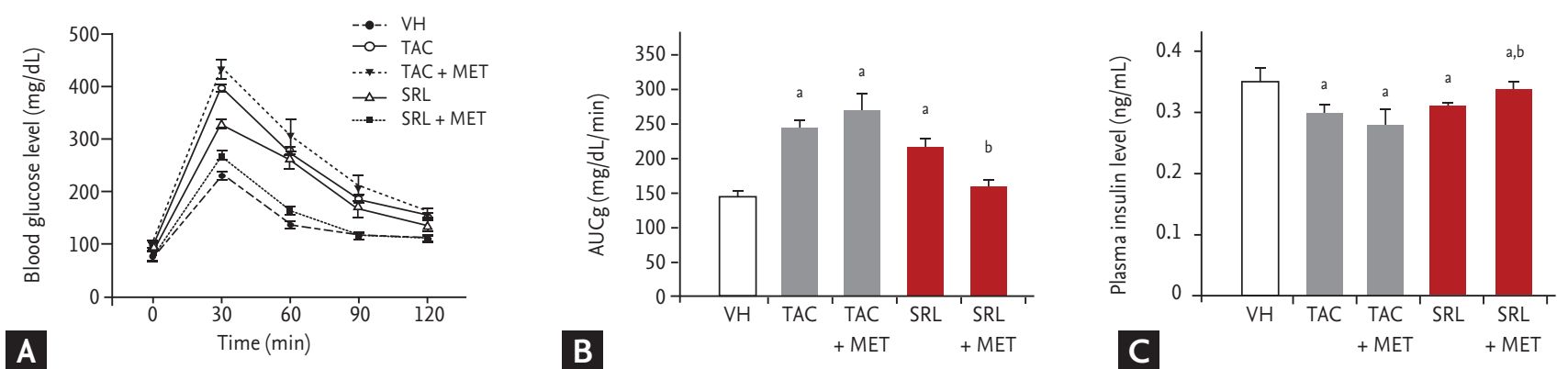

Figure 3. Effects of metformin (MET) on tacrolimus (TAC)- or sirolimus (SRL)-induced pancreatic islet dysfunction. (A) Intraperitoneal glucose tolerance test. (B) Area under the curve for glucose (AUCg). (C) Plasma insulin concentration. Note that the glucose and insulin concentrations were restored in the SRL + MET group compared with the SRL group but not in the TAC and TAC + MET groups. The results are expressed as the mean \pm SE of nine individual animals. VH, vehicle group. ${ }^{\mathrm{a}} \mathrm{p}<0.05 \mathrm{vs}$. $\mathrm{VH},{ }^{\mathrm{b}} \mathrm{p}<0.05$ vs. SRL.

antibody (1:100o; Cell Signaling Technology, Danvers, MA, USA) or polyclonal rabbit antiphosphorylated AMPK (p-AMPK, 1:1000; Cell Signaling Technology) diluted in SignalBoost immunoreaction enhancer (Millipore Corp.) as the primary antibody. The next day, the blots were incubated for 1 hour with peroxidase-conjugated goat antirabbit antibody (Cell Signaling Technology) diluted in SignalBoost immunoreaction enhancer. Antibody binding was detected using a commercial chemiluminescence kit (ATTO Corp., Tokyo, Japan). Quantification was performed using relative density, in which the VH group was assigned a density of $100 \%$ and the densities were normalized by each total AMPK band from the same gel (Quantity One version 4.4.0, Bio-Rad, Hercules, CA, USA).

\section{Statistical analysis}

Data are presented as mean \pm standard error. Significance between groups was determined using a one-way analysis of variance with the Bonferroni post hoc test
(SPSS version 19.0, IBM Co., Armonk, NY, USA). Significance was accepted at $p<0.05$.

\section{RESULTS}

\section{Effects of MET on basic parameters}

Table 1 shows the effect of MET with TAC or SRL on basic parameters in the experimental groups. The TAC and SRL groups had significantly lower body weight and higher 24-hour water intake and urine volume compared with the VH group. These changes were restored by treatment with MET. Serum creatinine concentration was higher in the TAC group, and lower in the TAC + MET group compared with the VH group. MET treatment did not affect the trough level of TAC or SRL in whole blood. 


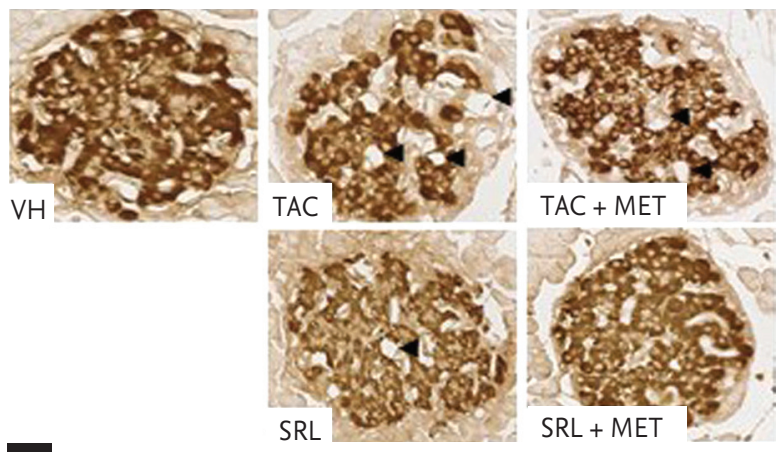

A

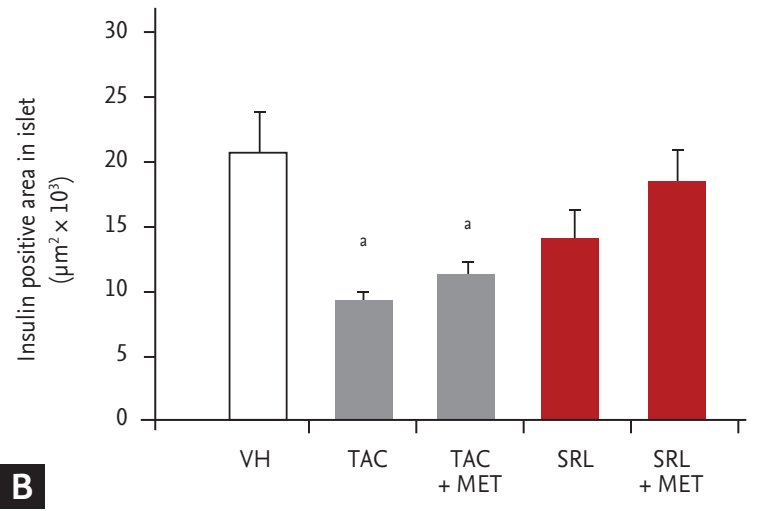

Figure 4. Effects of metformin (MET) on pancreatic islet size in tacrolimus (TAC)- or sirolimus (SRL)-induced pancreatic islet injury. (A) Immunostaining for insulin showing pancreatic islet morphology and size in the experimental group. Note that irregular islet boundaries and vacuolization (arrowheads) in the TAC and SRL groups. (B) Quantitative analysis of insulin-positive areas in islets. Note the significantly reduced islet size in the TAC and TAC + MET groups but not in the SRL and SRL + MET groups. The results are expressed as the mean $\pm \mathrm{SE}$ of nine individual animals. VH, vehicle group. ${ }^{\mathrm{a}} \mathrm{p}<0.05 \mathrm{vs}$. VH.

\section{Effects of MET on blood glucose and plasma insulin concentrations in diabetes caused by TAC or SRL}

The IPGT'T showed that TAC or SRL treatment for 4 weeks increased blood glucose concentration and decreased plasma insulin concentration (Fig. 3). The AUCg calculated from the IPGTT graph was higher in the TAC and SRL groups compared with the VH group. The combined treatment of MET for 2 weeks following induction of diabetes did not improve these changes in the TAC group. The AUCg was $244 \pm 7 \mathrm{mg} / \mathrm{dL} / \mathrm{min}$ in the TAC group and $270 \pm 19 \mathrm{mg} / \mathrm{dL} / \mathrm{min}$ in the TAC + MET group $(p<0.05)$. The insulin concentration was $0.30 \pm$ $0.01 \mathrm{ng} / \mathrm{mL}$ in the TAC group and $0.28 \pm 0.02 \mathrm{ng} / \mathrm{mL}$ in the TAC + MET group $(p>0.05)$. SRL induced hyperglycemia and reduced the plasma insulin concentration, but these effects were reversed by MET treatment. The AUCg was $216 \pm 8 \mathrm{mg} / \mathrm{dL} / \mathrm{min}$ in the SRL group and 160 $\pm 4 \mathrm{mg} / \mathrm{dL} / \mathrm{min}$ in the SRL + MET group $(p<0.05)$. The insulin concentration was $0.31 \pm 0.01$ in the SRL group and $0.34 \pm 0.01$ in the SRL + MET group $(p<0.05)$.

\section{Effects of MET on pancreatic islet size in TAC- and SRL-induced diabetes mellitus}

Immunostaining for insulin showed a strong and uniform pattern of staining in a large proportion of the islet $\beta$-cells in the VH group (Fig. 4A). However, a lower intensity of insulin staining, irregularity in islet boundaries, and evidence of vacuolization in islets were observed in $\beta$-cells from the TAC group. The SRL group showed similar insulin immunoreactivity to the VH group. Addition of MET did not change the staining intensity compared with TAC or SRL alone. Quantification of these results by measuring the insulin-positive areas (Fig. 4B) showed that the islet size was significantly smaller in the TAC and TAC + MET groups compared with the VH group $\left(9.2 \pm 0.9 \mu \mathrm{m}^{2} \times 10^{3}\right.$ in the TAC group and $11.4 \pm 0.9 \mu \mathrm{m}^{2} \times 10^{3}$ in the TAC + MET group vs. 20.7 $\pm 3.2 \mu \mathrm{m}^{2} \times 10^{3}$ in the $\mathrm{VH}$ group; $p<0.05$ compared with the $\mathrm{VH}$ group). The islet size did not differ significantly between the TAC and TAC + MET groups. Consistent with the insulin-staining results, islet size did not differ significantly between the VH, SRL, and SRL + MET groups $\left(14.1 \pm 2.2 \mu \mathrm{m}^{2} \times 10^{3}\right.$ in the SRL group and $18.4 \pm$ $2.5 \mu \mathrm{m}^{2} \times 10^{3}$ in the SRL + MET group).

\section{Effects of MET on GSIS in islets exposed to TAC or SRL} We used the GSIS test to confirm the in vivo results (Fig. 2). Exposure of isolated islets to TAC or SRL significantly reduced GSIS $(19.8 \pm 0.1 \mathrm{ng} / \mathrm{mL}$ in the TAC group and $20.2 \pm 0.1 \mathrm{ng} / \mathrm{mL}$ in the SRL group vs. $21.8 \pm 0.1 \mathrm{ng} / \mathrm{mL}$ in the VH group; $p<0.05$ vs. VH). Insulin concentration did not differ between the TAC + MET and TAC groups, whereas GSIS was restored in the SRL + MET group. GSIS was $20 \pm 0.1 \mathrm{ng} / \mathrm{mL}$ in the TAC+MET group $(p>$ 0.05 vs. the TAC group) and $21.1 \pm 0.2 \mathrm{ng} / \mathrm{mL}$ in the SRL + MET group ( $p<0.05$ vs. the SRL group) (Fig. 5 ). 


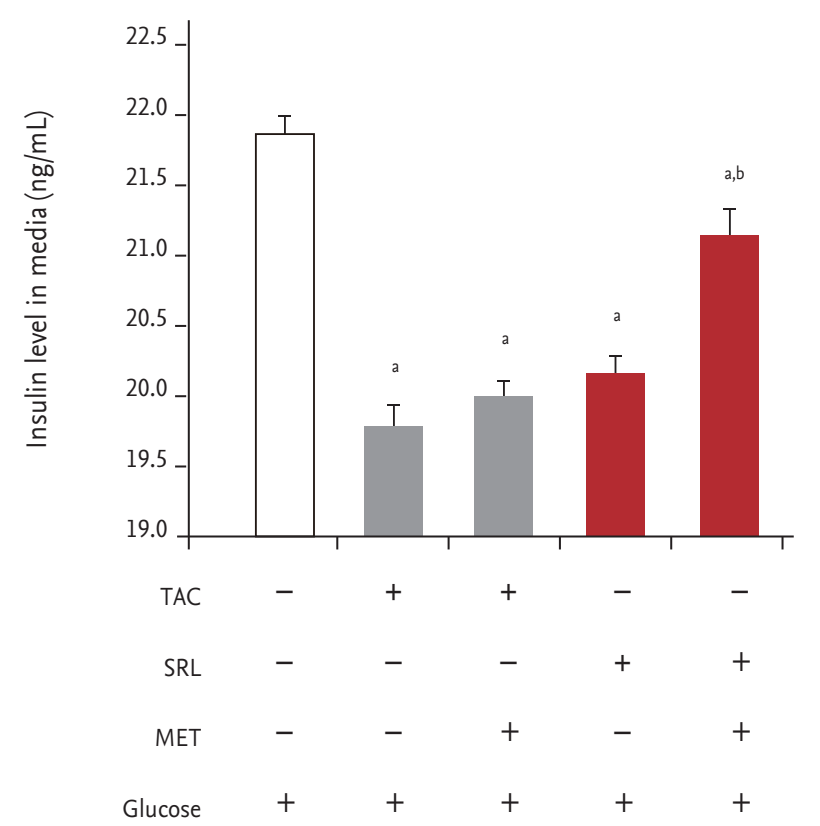

Figure 5. Direct effects of metformin (MET) on insulin secretion by tacrolimus (TAC) or sirolimus (SRL) assessed by the glucose-stimulated insulin secretion (GSIS) test. Insulin release in response to glucose was significantly lower in the TAC and SRL groups compared with the vehicle group. Note that the GSIS was significantly increased by MET combined with SRL but not by TAC compared with the corresponding non-MET-treated group. The results are expressed as the mean \pm SE of at least three independent experiments. ${ }^{\mathrm{a}} \mathrm{p}<$ 0.05 vs. VH, ${ }^{b} p<0.05$ vs. SRL.

\section{Effects of MET on AMPK expression in islets}

To evaluate the effects of MET on the expression of AMPK, a pharmacological target of MET, in TAC- and SRL-treated pancreatic islet cells, immunoblot analysis of $\mathrm{p}$-AMPK was conducted using the samples from the basal condition in the GSIS test (Fig. 6). The p-AMPK protein abundance relative to total AMPK abundance did not differ between the TAC + MET group compared with the TAC group. p-AMPK abundance was 1.6-fold lower in the SRL group compared with the $\mathrm{VH}$ group. By contrast, p-AMPK abundance was 2.4-fold higher in the SRL + MET group compared with the SRL group $(165 \% \pm 9 \%$ in the SRL + MET group vs. $67 \% \pm 2 \%$ in the SRL group, $p<0.05)$.

\section{DISCUSSION}

The present study was undertaken to investigate wheth-
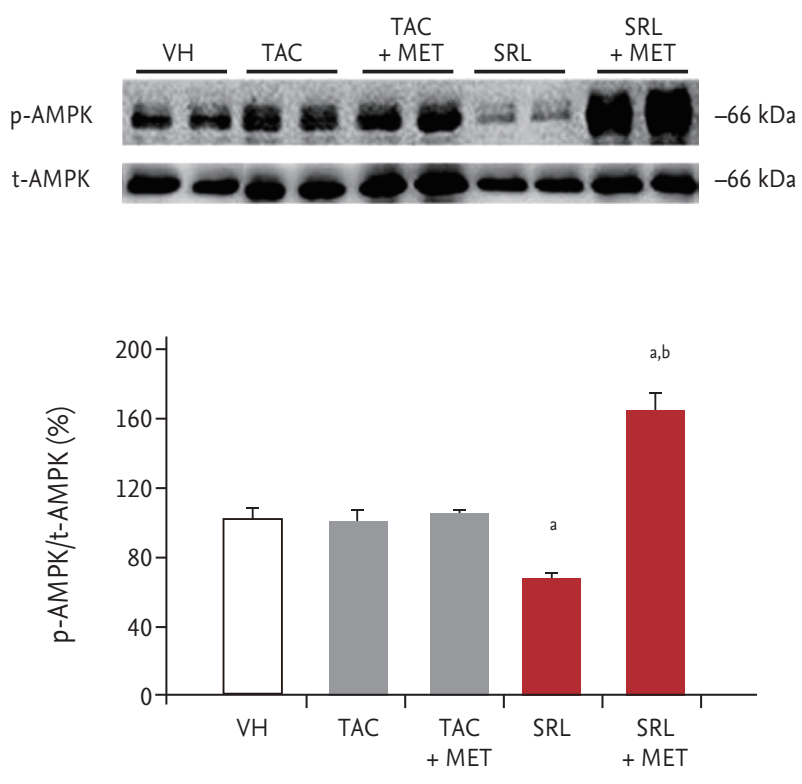

Figure 6. Effects of metformin (MET) on the expression of adenosine monophosphate-activated protein kinase (AMPK) during treatment with tacrolimus (TAC) or sirolimus (SRL). Representative immunoblot of phosphorylated ( $\mathrm{p}$-AMPK) and total AMPK (t-AMPK) in primary cultured rat islets treated with TAC or SRL with or without MET. The results of quantitative analysis are expressed as a ratio of $\mathrm{p}$-AMPK to t-AMPK. The density in the vehicle group (VH) was assigned a relative value of $100 \%$. The results are expressed as the mean \pm SE of at least three independent experiments. ${ }^{a} p$ $<0.05$ vs. VH, TAC, and TAC + MET, ${ }^{b}<0.05$ vs. SRL.

er MET, the first-line drug in the management of type 2 diabetes, is also effective in treating diabetes caused by immunosuppressant therapy. The results of the in vivo and in vitro experiments showed that MET was effective in altering the effects of SRL-induced diabetes but not those of TAC-induced diabetes. The differences in the effects of MET on diabetes seem to relate to the different causal mechanisms of diabetes. These results may be useful for understanding NODAT and when choosing antidiabetic drugs for patients with NODAT.

The most important finding of this study is that MET caused different glucose-lowering effects when combined with TAC- or SRL-induced hyperglycemia. Both drugs caused hyperglycemia and decreased plasma insulin concentration. However, the response to MET treatment differed between the TAC- and SRL-treated groups. In the SRL group, adding MET to SRL attenuated hyperglycemia and increased the plasma insulin 
concentration; this effect was not observed in the TAC group (Fig. 3). The results of our study are consistent with those of previous reports, which showed that MET treatment significantly reduced mean random glucose concentration in SRL-treated animals but not in TAC-treated animals [18].

It is generally accepted that insulin resistance is a main component to SRL-induced hyperglycemia. Therefore, it is expected that SRL induces hyperinsulinemia and adding MET to SRL decreases blood glucose and subsequent decline of serum insulin concentrations. On the contrary, our study showed that SRL treatment decreased plasma insulin level and adding MET to SRL increased plasma insulin level. This opposite result seems to related to the our model which is based on SRL-induced pancreatic islet cell injury, Thus, main pathogenesis of SRL-induced diabetes mellitus in our model is reduced insulin secretion rather than insulin resistance. This may explain why SRL treatment decreased plasma insulin level and adding MET to SRL increased plasma insulin level in our study.

In our study, immunohistochemical analysis of pancreatic islet cells showed that TAC decreased islet size, but SRL did not, and that MET treatment did not affect islet size in either group. This finding suggests that the decrease in plasma insulin concentration observed in the TAC and SRL groups is related to differences in the causal mechanisms of diabetes. In the TAC group, plasma insulin concentration decreased because of the reduced pancreatic $\beta$-cell mass, whereas in the SRL group, it decreased because of impaired insulin secretion. This conclusion is consistent with previous reports showing that the predominant mechanism of hyperglycemia induced by TAC is reduced $\beta$-cell mass through direct TAC-induced pancreatic $\beta$-cell injury and that hyperglycemia caused by SRL is closely associated with impaired insulin signaling [24].

To confirm the in vivo results showing different glucose-lowering effects of MET when combined with TAC or SRL, we performed the GSIS test (Fig. 2). Exposure of isolated islets to TAC or SRL significantly reduced GSIS. Combined SRL and MET treatment of islets restored GSIS (Fig. 5), whereas combined TAC and MET treatment did not. This finding suggests that MET improves insulin secretion in SRL-treated islets but not in TAC-treated islets and that the glucose-lowering effect of MET is mediated by increased insulin secretion in the SRL group.

AMPK is a key metabolic sensor in both insulin-sensitive and -insensitive tissues. It is well-known that antidiabetic properties of MET are mediated by activating the AMPK pathway in hepatocyte [25]. In our study, we found that MET also activates the AMPK expression in pancreatic islet cells, and this finding was consistent with previous reports that AMPK activator increase insulin release in isolated islets and the perfused pancreas [26] and activation of AMPK improves insulin sensitivity and increases insulin secretion in $\beta$-cells [27]. Taken together, our results suggests that MET may exert its glucose-lowering effect by activating the AMPK pathway in pancreatic islet cell, but further study will be needed to define a role of pancreatic islet compared to the hepatocyte in evaluating antidiabetic effect of MET.

The results of our study clearly demonstrate that SRL but not TAC treatment significantly decreased p-AMPK expression, and MET treatment significantly increased p-AMPK expression in SRL-treated but not in TAC-treated pancreatic islet cells. This finding suggests that SRL-induced diabetes is mediated by the AMPK pathway and that MET exerts its glucose-lowering effect by activating the AMPK pathway. It is interesting that that MET did not increase AMPK expression in TAC-treated pancreatic islet cells. We expected that MET increase AMPK expression because MET is a well known AMPK activator, but the effect of MET on AMPK activation was not significant in TAC-treated pancreatic islet cells. The reason for no increase of AMPK by MET is unclear, but we speculate two possibilities. First, AMPK signaling pathway may not be involved in TAC-induced pancreatic islet injury [28]. Second, MET dose may not be enough to activate AMPK in a setting of TAC-induced pancreatic islet injury.

The results of our study may have clinical applications. The current guideline for the management of NODAT is based on the treatment of type 2 diabetes, but the results of our study suggest that the choice of antidiabetic drugs in patients with NODAT should be made based on the type of immunosuppressant causing NODAT. In patients with TAC-induced diabetes, MET is not suitable as an antidiabetic drug for the control of hyperglycemia. Rather, dipeptidyl peptidase 4 inhibitors may be beneficial considering their protective effect against var- 
ious injuries [29]. Our results also suggest that MET may be recommended for patients with SRL-induced diabetes. This approach may provide a better rationale for the management of NODAT in KT recipients.

Our study has some limitations. First, 2 weeks of TAC or SRL treatment is too short to evaluate the long-term effect of each drug. Second, the trough level of TAC (around $10 \mathrm{ng} / \mathrm{mL}$ ) was higher and that of SRL (around $3.0 \mathrm{ng} / \mathrm{mL}$ ) was lower than the clinically recommended trough level. Third, we did not observe other beneficial effects of MET such as anti-inflammatory and antineoplastic effects [30,31].

In conclusion, the results of our study suggest that the development of NODAT differs according to the immunosuppressant used. The choice of antidiabetic drugs for NODAT should be made based on the mechanism through which immunosuppressant drugs cause diabetes.

\section{KEY MESSAGE}

1. The pathogenesis of new-onset diabetes after transplantation (NODAT) differs according to the type of immunosuppressant.

2. Antidiabetic drugs for NODAT should be chosen in accordance with the causal mechanism of NODAT.

\section{Conflict of interest}

No potential conflict of interest relevant to this article was reported.

\section{Acknowledgments}

This study was supported by a grant of the Korean Health Technology R\&D Project, Ministry for Health and Welfare, Republic of Korea (HI14C3417).

\section{REFERENCES}

1. Hjelmesaeth J, Flyvbjerg A, Jenssen T, et al. Hypoadiponectinemia is associated with insulin resistance and glucose intolerance after renal transplantation: impact of immunosuppressive and antihypertensive drug therapy. Clin J Am Soc Nephrol 2006;1:575-582.
2. Bee YM, Tan HC, Tay TL, Kee TY, Goh SY, Kek PC. Incidence and risk factors for development of new-onset diabetes after kidney transplantation. Ann Acad Med Singapore 2011;40:160-167.

3. Cosio FG, Pesavento TE, Kim S, Osei K, Henry M, Ferguson RM. Patient survival after renal transplantation: IV. Impact of post-transplant diabetes. Kidney Int 2002;62:1440-1446.

4. Luan FL, Steffick DE, Ojo AO. New-onset diabetes mellitus in kidney transplant recipients discharged on steroid-free immunosuppression. Transplantation 2011;91:334-341.

5. Rodrigo E, Fernandez-Fresnedo G, Valero R, et al. New-onset diabetes after kidney transplantation: risk factors. J Am Soc Nephrol 2006;17(12 Suppl 3):S291-S295.

6. Montori VM, Basu A, Erwin PJ, Velosa JA, Gabriel SE, Kudva YC. Posttransplantation diabetes: a systematic review of the literature. Diabetes Care 2002;25:583-592.

7. Israni AK, Zaun D, Rosendale JD, Snyder JJ, Kasiske BL. OPTN/SRTR 2012 annual data report: deceased organ donation. Am J Transplant 2014;14 Suppl 1:167-183.

8. Larson TS, Dean PG, Stegall MD, et al. Complete avoidance of calcineurin inhibitors in renal transplantation: a randomized trial comparing sirolimus and tacrolimus. Am J Transplant 2006;6:514-522.

9. Wullschleger S, Loewith R, Oppliger W, Hall MN. Molecular organization of target of rapamycin complex 2. J Biol Chem 2005;280:30697-30704.

10. Johnston O, Rose CL, Webster AC, Gill JS. Sirolimus is associated with new-onset diabetes in kidney transplant recipients. J Am Soc Nephrol 2008;19:1411-1418.

11. Yang SB, Tien AC, Boddupalli G, Xu AW, Jan YN, Jan LY. Rapamycin ameliorates age-dependent obesity associated with increased mTOR signaling in hypothalamic POMC neurons. Neuron 2012;75:425-436.

12. Ratner RE, Maggs D, Nielsen LL, et al. Long-term effects of exenatide therapy over 82 weeks on glycaemic control and weight in over-weight metformin-treated patients with type 2 diabetes mellitus. Diabetes Obes Metab 2006;8:419428.

13. Sharif A, Baboolal K. Complications associated with new-onset diabetes after kidney transplantation. Nat Rev Nephrol 2012;8:34-42.

14. Kurian B, Joshi R, Helmuth A. Effectiveness and longterm safety of thiazolidinediones and metformin in renal transplant recipients. Endocr Pract 2008;14:979-984.

15. Hwang H, Ghee JY, Song JH, Piao S, Yang CW. Compar- 
ison of adverse drug reaction profiles of two tacrolimus formulations in rats. Immunopharmacol Immunotoxicol 2012;34:434-442.

16. Song HK, Han DH, Song JH, et al. Influence of sirolimus on cyclosporine-induced pancreas islet dysfunction in rats. Am J Transplant 2009;9:2024-2033.

17. Cheng JT, Huang CC, Liu IM, Tzeng TF, Chang CJ. Novel mechanism for plasma glucose-lowering action of metformin in streptozotocin-induced diabetic rats. Diabetes 2006;55:819-825.

18. Shivaswamy V, Bennett RG, Clure CC, Larsen JL, Hamel FG. Metformin improves immunosuppressant induced hyperglycemia and exocrine apoptosis in rats. Transplantation 2013;95:280-284.

19. Choi YH, Kim SG, Lee MG. Dose-independent pharmacokinetics of metformin in rats: hepatic and gastrointestinal first-pass effects. J Pharm Sci 2006;95:2543-2552.

20. Quaile MP, Melich DH, Jordan HL, et al. Toxicity and toxicokinetics of metformin in rats. Toxicol Appl Pharmacol 2010;243:340-347.

21. Jin L, Lim SW, Doh KC, et al. Dipeptidyl peptidase IV inhibitor MK-0626 attenuates pancreatic islet injury in tacrolimus-induced diabetic rats. PLoS One 2014;9:e100798.

22. Lim SW, Jin L, Piao SG, Chung BH, Yang CW. Inhibition of dipeptidyl peptidase IV protects tacrolimus-induced kidney injury. Lab Invest 2015;95:1174-1185.

23. Piao SG, Bae SK, Lim SW, et al. Drug interaction between cyclosporine and mTOR inhibitors in experimental model of chronic cyclosporine nephrotoxicity and pan- creatic islet dysfunction. Transplantation 2012;93:383-389.

24. Shivaswamy V, Bennett RG, Clure CC, et al. Tacrolimus and sirolimus have distinct effects on insulin signaling in male and female rats. Transl Res 2014;163:221-231.

25. Pernicova I, Korbonits M. Metformin: mode of action and clinical implications for diabetes and cancer. Nat Rev Endocrinol 2014;10:143-156.

26. Akkan AG, Malaisse WJ. Insulinotropic action of AICA riboside. I. Insulin release by isolated islets and the perfused pancreas. Diabetes Res 1994;25:13-23.

27. Leclerc I, Viollet B, da Silva Xavier G, Kahn A, Rutter GA. Role of AMP-activated protein kinase in the regulation of gene transcription. Biochem Soc Trans 2002;30:307-311.

28. Saeedi R, Parsons HL, Wambolt RB, et al. Metabolic actions of metformin in the heart can occur by AMPK-independent mechanisms. Am J Physiol Heart Circ Physiol 2008;294:H2497-H2506.

29. Lim SW, Jin JZ, Jin L, Jin J, Li C. Role of dipeptidyl peptidase-4 inhibitors in new-onset diabetes after transplantation. Korean J Intern Med 2015;30:759-770.

30. Jeong YK, Kim MS, Lee JY, Kim EH, Ha H. Metformin radiosensitizes p53-deficient colorectal cancer cells through induction of $\mathrm{G} 2 / \mathrm{M}$ arrest and inhibition of DNA repair proteins. PLoS One 2015;10:e0143596.

31. Jang EK, Kim WG, Kwon H, et al. Metformin is associated with a favorable outcome in diabetic patients with cervical lymph node metastasis of differentiated thyroid cancer. Eur Thyroid J 2015;4:181-188. 